\title{
Factores asociados a infecciones respiratorias dentro de los tres primeros meses de vida
}

\author{
R. MAURICIO BARRÍA P. ${ }^{1}$, MARIO CALVO G. ${ }^{2}$
}

1. Enfermero, M.Sc. Epidemiología Clínica. Académico, Instituto de Enfermería, Facultad de Medicina, Universidad Austral de Chile. Becario Proyecto Mecesup AUS 0209, Programa de Doctorado en Salud Pública, Escuela de Salud Pública, Universidad de Chile.

2. Médico Pediatra, Profesor Titular Instituto de Pediatría y Decano de la Facultad de Medicina, Universidad Austral de Chile.

\begin{abstract}
Factors associated to morbidity consultations due respiratory infection within first three months of life
\end{abstract}

Background: Acute respiratory infections (ARI) are the most frequent cause of medical consultation in primary care, emergency room, as well as, for pediatric hospitalization. Objective: To characterize the morbidity by ARI until the third month of life and to evaluate its risk factors. Methods: Cohort study in fullterm newborns $(n=316)$ admitted with their mothers at the Maternity of the Hospital Clinico Regional of Valdivia. During 3 months of follow-up, medical consultations by ARI were identified, and overall and specific incidence for ARI was estimated. Maternal, neonatal, socioeconomic and environmental factors were evaluated as associated factors with lower respiratory tract infections (LRTI), using stepwise logistic regression. Results: The overall incidence rate of ARI was 20.4 episodes per 100 child-months, while LRTI reached 6.2 episodes per 100 child-months. Incidence rate of common cold, wheezing bronchitis and pneumonia was $11.6,4.4$ and 0.85 per 100 child-months, respectively. Risk factors for LRTI were: unmarried single mother [ $\left.\mathrm{OR}=2.6 ; \mathrm{CI}_{95 \%} 1.22-5.60\right]$, indoor tobacco smoke [OR=2.9; $\mathrm{CI}_{95 \%}$ 1.34-6.33], use of wood as exclusive fuel [OR=4.0; $\mathrm{CI}_{95 \%}$ 1.66-9.79] and season (March-July) [OR=7.6; $\mathrm{CI}_{95 \%}$ 3.25-18.0]. Conclusions: ARI persists as main cause of ambulatory medical consultation in smaller babies, and environmental factors acquire fundamental importance for prevention in its occurrence.

(Key words: infant, medical consultations, morbidity, newborn, respiratory tract infections, risk factors) Rev Chil Pediatr 2008; 79 (3): 281-289

\section{RESUMEN}

Introducción: Las infecciones respiratorias agudas (IRA) constituyen el principal motivo de consultas en centros primarios y de urgencia, así como de hospitalización pediátrica. Objetivos: Caracterizar la morbilidad

Trabajo recibido el 07 de abril de 2008, devuelto para corregir el 29 de abril de 2008, segunda versión el 23 de mayo de 2008, aceptado para publicación el 26 de mayo de 2008.

Fuente de Financiamiento: Estudio financiado parcialmente por la Dirección de Investigación de la Universidad Austral de Chile (Proyecto DID S-2005-43).

Correspondencia a:

Mauricio Barría P.

E-mail: rbarria@uach.cl 
por IRA hasta el tercer mes de vida y evaluar sus factores de riesgo. Métodos: Cohorte de recién nacidos

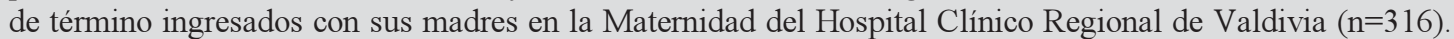
Durante 3 meses de seguimiento se estableció la morbilidad por IRA y estimó la tasa de incidencia global y específica. La asociación entre IRA y factores maternos-neonatales, socioeconómicos y ambientales se evaluó mediante regresión logística stepwise. Resultados: La incidencia de IRA global e IRA baja (IRAB) fue 20,4 y 6,2 episodios por cien meses-niño, respectivamente. La tasas por diagnósticos fueron: resfrío 11,6, síndrome bronquial obstructivo 4,4 y neumonía 0,85 episodios por cien meses-niño. Los factores de riesgo para IRAB fueron: ser hijo de madre soltera (sin pareja) [OR=2,6; $\left.\mathrm{IC}_{95 \%} 1,22-5,60\right]$, tabaquismo intradomiciliario $\left[\mathrm{OR}=2,9 ; \mathrm{IC}_{95 \%} 1,34-6,33\right]$, uso exclusivo de leña como combustible $\left[\mathrm{OR}=4,0 ; \mathrm{IC}_{95 \%} 1,66\right.$ 9,79] y estacionalidad (Marzo-Julio) [OR=7,6; $\mathrm{IC}_{95 \%}$ 3,25-18,0]. Conclusiones: Las IRA persisten como principal motivo de consulta ambulatoria en lactantes pequeños, en quienes los factores ambientales adquieren vital importancia para su prevención.

(Palabras clave: consulta médica, morbilidad, factor de riesgo, infección respiratoria aguda, lactante, recién nacido).

Rev Chil Pediatr 2008; 79 (3): 281-289

\section{Introducción}

Las infecciones respiratorias agudas (IRA) son un importante problema de salud pública, siendo reconocidas como la principal causa de morbilidad pediátrica y más frecuente motivo de utilización de los servicios de salud. Las estimaciones muestran que las IRA representan entre 30 y $50 \%$ de las visitas de los niños a los establecimientos de salud y entre 20 y $40 \%$ de las hospitalizaciones pediátricas en la mayoría de los países en vías de desarrollo ${ }^{1}$. Específicamente en Chile, se ha observado que las IRA concentran más de $67 \%$ de las consultas de menores de seis años en atención prima$\mathrm{ria}^{2}$, en tanto que a nivel de servicios de urgencia se ha constatado que, del total de consultas pediátricas, los diagnósticos más frecuentemente consignados corresponden a IRA alta (IRAA) y síndrome bronquial obstructivo (SBO) ${ }^{3}$. En particular, de las afecciones respiratorias, el SBO y la neumonía son consideradas graves y han abocado un interés específico por originar hospitalizaciones, gasto de recursos sanitarios, predisposición a enfermedades crónicas e incluso ocasionar la muerte ${ }^{4}$. Dentro de las IRA bajas (IRAB), el SBO concentra entre 23 y $25 \%$ constituyéndose en la principal causa específica de morbilidad pediátrica. La neumonía por su parte, llega a $2,1 \%$ pero es la principal causa de mortalidad infantil tardía, superando el $90 \%$ de las muertes por IRA ${ }^{5}$.

Mientras las IRAA corresponden a las en- fermedades más comunes de la infancia temprana, las IRAB explican la mayoría de las afecciones severas o crónicas ${ }^{6} \mathrm{y}$ aunque persisten como preocupación durante toda la infancia, se ha confirmado que la incidencia de SBO y neumonía disminuye con la edad, y particularmente es alta en menores de una año ${ }^{4}$. De esta manera, parece lógico evaluar la magnitud del problema en aquellos niños biológicamente más vulnerables y en donde la severidad se prevé mayor. Así por ejemplo, ser menor de 3 meses se ha considerado un importante factor de riesgo de hospitalización por bronquiolitis ${ }^{7}$. Por su parte, los factores de riesgo de IRA y en particular de IRAB, han sido ampliamente estudiados, asociándose significativamente con factores sociodemográficos (edad materna, escolaridad de los padres, nivel socioeconómico) y ambientales (contaminación del aire, hacinamiento, temperatura y humedad, entre otros) $)^{8}$.

En este escenario, el objetivo del estudio fue caracterizar las consultas de morbilidad espontáneas (CME) por IRA dentro de los tres primeros meses de vida y evaluar sus factores de riesgo asociados.

\section{Pacientes y Métodos}

Estudio de cohorte prospectiva en recién nacidos (RN) sanos con peso de nacimiento $(\mathrm{PN}) \geq 2500 \mathrm{~g}$ y edad gestacional $(\mathrm{EG}) \geq 37$ 
semanas, transferidos junto a sus madres desde la unidad de partos a la Maternidad del Hospital Clínico Regional de Valdivia, Chile. Este estudio fue parte de un proyecto de investigación para detectar predictores de hospitalización al tercer mes de vida, y fue presentado y aprobado por el Comité Ético Científico institucional y la dirección del Servicio de Obstetricia y Ginecología del hospital. Además, requirió del consentimiento libre, informado y por escrito por parte de las madres.

Entre Octubre de 2005 y Julio de 2006 se invitó a participar a las madres cuyos hijos cumplían los criterios de elegibilidad. Además de la condición de PN y EG se estableció como límite geográfico tener residencia en la ciudad de Valdivia. Se excluyeron los niños con puntuación apgar $<7$ al minuto, con malformación congénita, en condición de abandono y aquellos nacidos de parto gemelar (por no constituir observaciones independientes). Adicionalmente, fueron excluidos los neonatos que desde el nacimiento requirieron hospitalización. De 386 RN elegibles, 14 madres rehusaron participar conformándose finalmente una cohorte de 372 niños.

Dentro de las primeras 48 horas posteriores al parto se presentó el estudio a las madres y obtuvo el consentimiento para su incorporación. Posterior a ello, se revisaron los expedientes clínicos maternos (ficha clínica, carné de control prenatal) y del neonato (hoja de parto y evaluación pediátrica), aplicándose además un cuestionario por parte de un entrevistador.

Se estableció como resultado principal la consulta de morbilidad por IRA. Esta se definió como la asistencia espontánea ambulatoria a un centro de salud público o privado (consultorio, centro de salud familiar, urgencia pediátrica, consulta privada) adicional a los controles programados como control de salud infantil. No se consideraron tampoco los controles subsecuentes a una consulta previa, asumiéndose de esta forma, cada consulta como un nuevo episodio de enfermedad.

Se consignaron el motivo principal y lugar de las consultas. De acuerdo a los diagnósticos encontrados, se clasificaron las IRA en altas y bajas distribuyendo las afecciones de la siguiente manera:
- IRA alta: resfrío, virosis respiratoria alta (VRA), faringitis, rinofaringitis.

- IRA baja: síndrome coqueluchoideo, bronquitis aguda, SBO, neumonía.

Se analizaron antecedentes maternos, neonatales, familiares y ambientales. De las madres se recopilaron datos sociodemográficos (edad, escolaridad, estado civil, actividad) y obstétricos (paridad, lapso intergestacional, programación del embarazo, control prenatal, patología del embarazo, consumo de alcohol, cigarro o drogas durante el embarazo, tipo de parto). Del $\mathrm{RN}$ se consideraron las variables $\mathrm{PN}, \mathrm{EG}$, sexo, puntuación Apgar. Los antecedentes familiares estudiados fueron tamaño del grupo familiar, número de hijos, asistencia de niños a jardín infantil o sala cuna. Se consignó el ingreso económico familiar mensual informado por la madre. Con ello, se calculó el ingreso per cápita dividiendo ese valor por el número de integrantes del grupo familiar.

Desde el punto de vista ambiental interesaron las características de la vivienda, número de habitaciones y dormitorios, tipo de calefacción, tipo de combustibles regularmente empleados y tabaquismo intradomiciliario (TID). Para ponderar el efecto estacional sobre el desarrollo de IRA, se generó la variable "estacionalidad" categorizándola de acuerdo al periodo en que los niños nacieron y desarrollaron el seguimiento. Así, aquellos nacidos entre octubre y febrero y cuyo seguimiento no sobrepaso el mes de mayo, fueron considerados de menor riesgo, en comparación con los nacidos entre marzo y julio.

Las madres fueron contactadas por teléfono y/o visita domiciliaria hasta cumplirse 3 meses postparto constatándose las CME. Se consignó igualmente las características de la lactancia materna (LM) y verificó si alguna de las condiciones sociodemográficas o ambientales habían sido modificadas en el periodo de seguimiento.

Los datos fueron analizados con estadística descriptiva usando distribución de frecuencias, medidas de tendencia central y de dispersión. Las variables categóricas y nominales como sexo del RN, tipo de parto, estado civil, escolaridad materna, etc. se describen como propor- 
ción. Por su parte, las variables continuas u ordinales como edad materna, PN, EG, y puntuación Apgar al primer y quinto minuto se presentan como media y desviación estándar ( $\pm \mathrm{DE})$ o mediana y rango (min-máx) o rango intercuartil [p25-p75], cuando la distribución no fue normal. Se focalizó en la morbilidad por IRAB, comparándose el comportamiento de las variables entre los niños que desarrollaron y no desarrollaron este evento. Para esto, se evaluó asociación univariada mediante la prueba exacta de Fisher para datos categóricos y la prueba de la t de Student (t-test) o de MannWhitney para variables numéricas continuas, según correspondió. Se calculó la tasa de incidencia global de consultas de morbilidad y por los principales diagnósticos de consulta expresada como eventos por cien meses-niño. Se calculó razones de tasas de incidencia (RTI) y su intervalo de confianza $95 \%\left(\mathrm{IC}_{95}\right)$ para comparar variables Conforme al breve periodo de seguimiento en el cual hay una baja probabilidad de desarrollar más de un evento de IRAB como para emplear otras técnicas estadísticasse optó como aproximación multivariada por la regresión logística múltiple obteniéndose factores asociados significativamente y de manera independiente a IRAB como evento dicotómico (OR ajustados). Las variables analizadas fueron aquellas que en la estimación univariada mostraron asociación a un valor $\mathrm{p}<0,1$. Se empleó la técnica stepwise con un nivel de permanencia de 0,05 . El procesamiento y análisis de los datos fueron realizados con el programa Stata versión 8.1 (Stata Corporation, College Station, Texas, USA 2003).

\section{Resultados}

\section{Características generales}

De los 372 RN incorporados inicialmente, se logró obtener información completa al tercer mes de 316 niños, perdiéndose el contacto de 56 niños en las primeras semanas (figura 1). Además, ocurrió el fallecimiento de un niño cuyo diagnóstico fue consignado como síndrome de muerte súbita a los 26 días de vida. No se observaron diferencias estadísticamente significativas en las características basales maternas y neonatales entre los que completaron el seguimiento y los que no.

El PN y EG de la cohorte estudiada fue $3516 \pm 410$ g y $39,2 \pm 1,0$ semana, respectivamente, siendo $6,7 \%$ considerados pequeños para la edad gestacional (PEG). Nacieron por cesárea $29,8 \%$ de los niños. De éstas, 47,9\% (45/94) fueron consideradas cesáreas de urgencia. La puntuación media de Apgar fue 8,9 \pm 0,35 al minuto y $9,4 \pm 0,49$ a los cinco. Del total de nacimientos, $44,9 \%$ ocurrieron en el periodo definido como de mayor riesgo (marzo-julio).

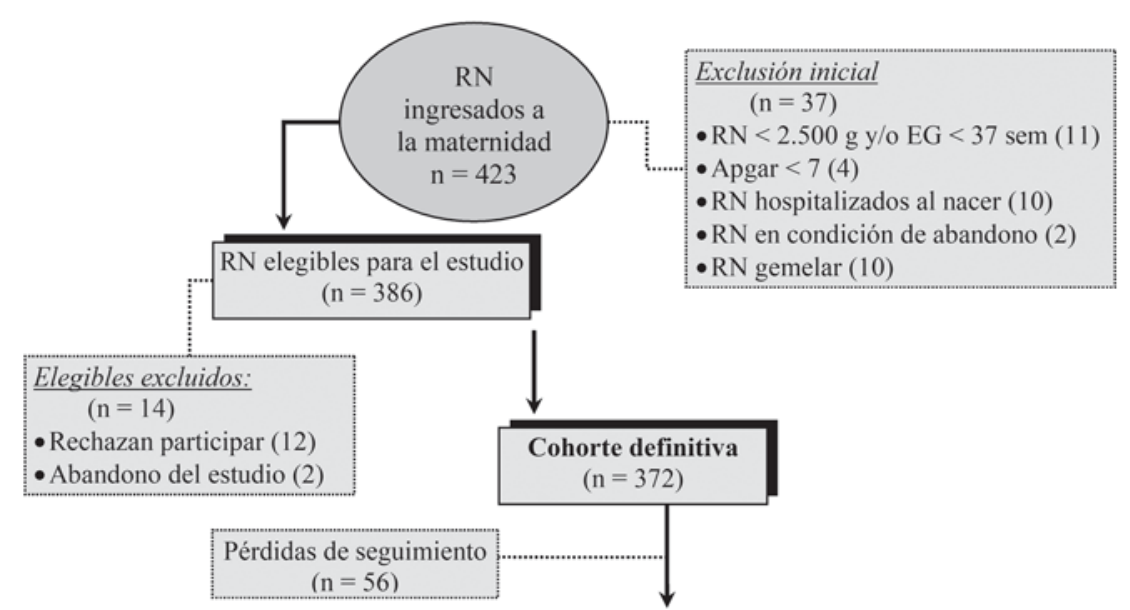

Analizados $(\mathrm{n}=316)$

Figura 1. Diagrama del estudio. 
Tabla 1. Distribución de niños consultantes por IRA e IRAB según número de visitas efectuadas

\begin{tabular}{|c|c|c|c|c|}
\hline \multirow[t]{2}{*}{ Visitas } & \multicolumn{2}{|c|}{$\begin{array}{c}\text { consultantes } \\
\text { por IRA } \\
n=128\end{array}$} & \multicolumn{2}{|c|}{$\begin{array}{c}\text { consultantes } \\
\text { por IRAB } \\
n=43\end{array}$} \\
\hline & $n$ & $\%$ & $\mathbf{n}$ & $\%$ \\
\hline 1 & 83 & 64,8 & 30 & 69,8 \\
\hline 2 & 31 & 24,2 & 10 & 23,2 \\
\hline 3 & 9 & 7,0 & 3 & 7,0 \\
\hline 4 & 4 & 3,1 & 0 & 0 \\
\hline 5 & 1 & 0,8 & 0 & 0 \\
\hline
\end{tabular}

Las edad mediana de las madres fue de 23 años (rango 14-43). Se consignó 26,3\% de embarazo adolescente $(<20$ años) y $55,7 \%$ de embarazo no planificado. Como era de esperar, esta última condición fue significativamente mayor en mujeres adolescentes $(69,9 \%$ vs $50,6 \%, p=0,003)$. La primiparidad fue de $47,1 \%$. El número de CPN tuvo una mediana de 11 [913]. El consumo de alcohol y cigarrillos en algún momento del embarazo fue señalado por 15,8 y $22,8 \%$ respectivamente.

La distribución por estado civil/conyugal de las madres mostró una proporción similar de convivientes $(35,8 \%)$ y solteras sin pareja actual (34,2\%). El número de hijos mediano fue 2 (rango: 1-7). La mediana de integrantes del grupo familiar fue 5 [4-6]. Se estimó un ingreso familiar mensual per cápita mediano de $\$ 32000$ [24 000-50 000]. La mayoría (46,9\%) ubicado en el tramo de $\$ 20000$ a $\$ 40000$ y 17,5\% bajo ese rango.

En relación a condiciones ambientales, 98,7\% tenía acceso a agua potable, y 89,2\% disponía de baño interior. Se encontró en $23,7 \%$ de los hogares una distribución de al menos tres personas por dormitorio y en $9,5 \%$ de cuatro o más. En 18,3\% de los hogares se observó la asistencia de otro hijo o niño a jardín infantil o sala cuna. Se consignó $89,9 \%$ de madres quienes declararon uso de leña como medio de calefacción y/o cocción de alimentos, siendo empleado como combustible exclusivo en $64,2 \%$ de los casos. La prevalencia de hábito tabáquico en algún miembro del hogar fue 44,6\%, mientras que el TID alcanzó 26,6\%. Se estableció que al término del tercer mes $69,8 \%$ de las madres mantenían LM exclusiva (LME).

\section{Características de la morbilidad respiratoria}

El período de seguimiento aportado por los integrantes de la cohorte consideró 946 meses a riesgo de enfermar. Globalmente, 198 niños $(62,6 \%)$ realizaron CME sumando 322 visitas. Específicamente fueron diagnosticados de alguna IRA 128 niños (40,5\% del total y 64,6\% de los consultantes), generándose 193 consultas por este motivo $(59,9 \%)$. Se observó que 83 de estos lactantes $(64,8 \%)$ efectuaron una visita única y pocos casos a esta edad (14) consultaron más de 2 veces (tabla 1). Los lactantes afectados por IRAB fueron $43(13,6 \%)$ los que sumaron 59 consultas. Más de la mitad de las consultas se realizaron en un consultorio o CESFAM (50,3\%), mientras $37,3 \%$ se efectuaron en la urgencia pediátrica. Asimismo, y a pesar que la población estudiada era beneficiaria del sistema público, un $12,4 \%$ acudió al sistema privado.

Los diagnósticos más frecuentes fueron resfrío y SBO con 57 y $21,8 \%$, respectivamente (figura 2). La tasa global de consultas por IRA alcanzó 20,4 por cien meses-niño y específicamente para resfrío 11,6 por cien meses-niño. Las IRAB en tanto mostraron una tasa de 6,2 eventos por cien meses-niño. Las 42 consultas por SBO fueron generadas por 29 niños $(9,2 \%)$, alcanzando así una tasa de 4,4 episodios por cien meses-niño. Los 8 casos de neumonía diagnosticados durante el seguimiento determinaron una tasa de 0,85 por cien meses-niño.

Se establecieron diferencias estadísticamente significativas en las tasas de incidencia de IRAB con la presencia de TID [RTI $=2 ; \mathrm{IC}_{95}$ 1,03$3,82]$, en los nacidos entre marzo y julio [RTI $=$ 4,03; IC $\left._{95} 1,94-9,16\right]$, en hogares con uso exclusivo de leña $\left[\mathrm{RTI}=2,1 ; \mathrm{IC}_{95} 0,99-5,0\right]$ y en hijos de madres que fumaron durante el embarazo [RTI $=2 ;$ IC $_{95}$ 1,01-3,85]. Consecuentemente, la comparación univariada entre niños que consultaron por IRAB y los que no, evidenció diferencias estadísticamente significativas para las variables consumo de cigarrillo en el embarazo, TID, estacionalidad y uso exclusivo de leña (tabla 2).

Como estimación ajustada del riesgo de 
IRAB (OR ajustado), el análisis multivariado reveló que los factores asociados significativamente a un incremento del riesgo de consultar

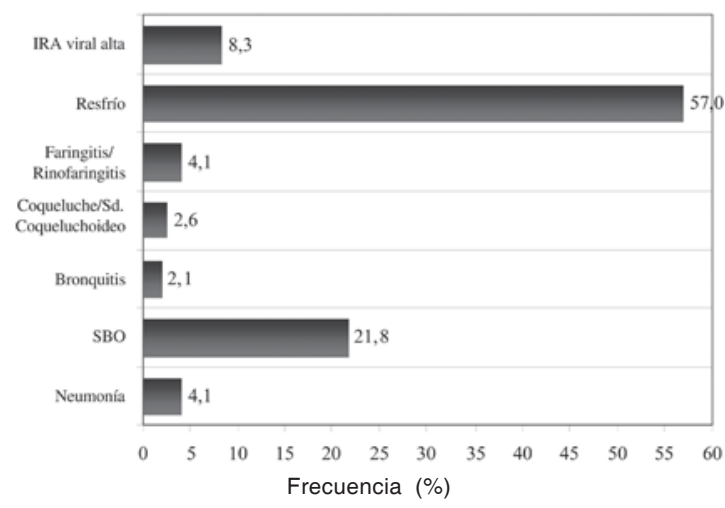

Figura 2. Distribución de las consultas de morbilidad por IRA según diagnóstico. por dicha causa fueron ser hijo de madre soltera (sin pareja actual), la estacionalidad, el TID y el uso exclusivo de leña (tabla 3 ).

\section{Tabla 3. Factores significativamente asociados a consulta de morbilidad espontánea por IRAB al tercer mes de vida}

\begin{tabular}{lccc}
\hline Variable* & $\begin{array}{c}\text { OR } \\
\text { Ajustado }\end{array}$ & $\begin{array}{c}\text { Intervalo de } \\
\text { confianza } \\
\mathbf{9 5 \%}\end{array}$ & $\mathbf{p}$ \\
\hline Soltera & 2,6 & $1,22-5,60$ & 0,013 \\
TID & 2,9 & $1,34-6,33$ & 0,007 \\
Uso exclusivo de leña & 4,0 & $1,66-9,79$ & 0,002 \\
Estacionalidad & & & \\
(Marzo-Julio) & 7,6 & $3,25-18,0$ & 0,000 \\
\hline
\end{tabular}

TID: tabaquismo intradomiciliario. *Usando regresión logística stepwise fueron removidas del modelo en pasos sucesivos las variables consumo de alcohol en el embarazo $(p=0,31)$, consumo de tabaco en el embarazo $(p=0,61)$, edad gestacional $(p=0,06)$, y embarazo adolescente $(0,28)$.

Tabla 2. Características de la cohorte según presencia/ausencia de IRAB

\begin{tabular}{|c|c|c|c|}
\hline Variable & $\begin{array}{l}\text { IRAB (-) } \\
n=273\end{array}$ & $\begin{array}{c}\text { IRAB (+) } \\
n=43\end{array}$ & $\mathbf{P}$ \\
\hline $\begin{array}{l}\text { Características maternas y del embaraz } \\
\text { Edad materna (años), Mediana [RIC] } \\
\text { Madre adolescente (\%) } \\
\text { Escolaridad básica completa (\%) } \\
\text { Estado civil soltera (\%) } \\
\text { Embarazo no programado (\%) } \\
\text { Control Prenatal (n) Mediana [RIC] } \\
\text { Consumo de alcohol en el embarazo (\%) } \\
\text { Consumo de cigarrillo en el embarazo (\%) } \\
\text { Primiparidad (\%) } \\
\text { LME al tercer mes (\%) }\end{array}$ & $\begin{array}{c}23[20-29,5] \\
24,5 \\
94,5 \\
32,2 \\
55,7 \\
11[9-13] \\
14,3 \\
20,5 \\
45,8 \\
70,6\end{array}$ & $\begin{array}{c}21[18-29] \\
37,2 \\
90,7 \\
46,5 \\
55,8 \\
12[9-13] \\
25,6 \\
37,2 \\
55,3 \\
65,1\end{array}$ & $\begin{array}{l}0,186^{\ddagger} \\
0,093^{*} \\
0,306^{*} \\
0,083^{*} \\
1,000^{*} \\
0,677^{\ddagger} \\
0,072^{*} \\
0,019^{*} \\
0,252^{*} \\
0,478^{*}\end{array}$ \\
\hline $\begin{array}{l}\text { Características del RN y parto } \\
\text { PN (g) Media } \pm \mathrm{DE} \\
\text { EG (semanas) Media } \pm \mathrm{DE} \\
\text { PEG (\%) } \\
\text { Apgar } 1 \text { minuto Media } \pm \mathrm{DE} \\
\text { Apgar } 5 \text { minutos Media } \pm \mathrm{DE} \\
\text { Sexo masculino (\%) } \\
\text { Cesárea (\%) }\end{array}$ & $\begin{array}{c}3526 \pm 413 \\
39,3 \pm 1,01 \\
7,0 \\
8,9 \pm 0,37 \\
9,3 \pm 0,48 \\
48,7 \\
28,6\end{array}$ & $\begin{array}{l}3451 \pm 389 \\
38,9 \pm 1,03 \\
4,6 \\
9 \quad \pm 0,22 \\
9,4 \pm 0,49 \\
62,8 \\
37,2\end{array}$ & $\begin{array}{l}0,266^{\dagger} \\
0,076^{\dagger} \\
0,750 \\
0,157^{\dagger} \\
0,534^{\dagger} \\
0,101^{*} \\
0,282^{*}\end{array}$ \\
\hline $\begin{array}{l}\text { Características familiares y ambientales } \\
\text { Tamaño grupo familiar (n) Mediana [RIC] } \\
\text { Ingreso familiar per capita < } \$ 25.000(\%) \\
\text { Tres o más personas por dormitorio (\%) } \\
\text { Asistencia de otro hijo a jardín infantil (\%) } \\
\text { Estacionalidad Marzo - Julio (\%) } \\
\text { Uso exclusivo de leña (\%) } \\
\text { Tabaquismo intradomiciliario (\%) }\end{array}$ & $\begin{array}{l}5[4-6] \\
35,1 \\
23,4 \\
18,3 \\
39,9 \\
61,9 \\
24,2\end{array}$ & $\begin{array}{l}5[4-6] \\
37,2 \\
25,6 \\
18,6 \\
76,7 \\
79,1 \\
41,9\end{array}$ & $\begin{array}{l}0,304^{\ddagger} \\
0,864^{*} \\
0,847^{*} \\
1,000^{*} \\
0,000^{*} \\
0,039^{*} \\
0,024^{*}\end{array}$ \\
\hline
\end{tabular}

* Test exacto de Fisher. ${ }^{\dagger}$ Test $t$ de Student. ${ }^{\ddagger}$ Test de Mann-Whitney.

RIC: rango intercuartil. DE: desviación estándar. LME: lactancia materna exclusiva. PN: peso de nacimiento. EG: edad gestacional. PEG: pequeño para la edad gestacional. 


\section{Discusión}

En nuestro estudio, la morbilidad respiratoria fue el principal motivo de las CME a nivel primario en niños sanos al nacer. Si bien las IRAA concentran el mayor número de visitas, las IRAB, y en particular el SBO, aportan sustancialmente en la estructura de la morbilidad respiratoria hasta el tercer mes de vida.

Aunque no son estrictamente comparables, las cifras reportadas por López et al, en seguimientos más prolongados han dado cuenta de que las IRA han concentrado $67 \%$ del total de consultas efectuadas ambulatoriamente por menores de 6 años observados por un año ${ }^{2}$ y de las consultas generadas por lactantes hasta el año y medio ${ }^{9}$. En este último estudio, dentro de los seis primeros meses de vida se registró una incidencia global de IRA de 38,3 episodios por cien meses-niño en tanto que para IRAB alcanzó 17,5 por cien meses-niño. Pino et al, evaluaron una cohorte de 504 niños a partir del cuarto mes de vida estimando una incidencia global de IRAB de 28 eventos por cien mesesniño. En este estudio, la tasa de incidencia específica para bronquitis, bronquiolitis y SBO fue respectivamente $4,8,0,4$ y 19,5 por cien meses-niño ${ }^{10}$. Koch et al, registraron tasas de incidencias de 35,5 y 17,3 episodios por cien meses-niño de IRA e IRAB, respectivamente hasta el quinto mes. Se observó además tres veces mayor riesgo de $\mathrm{IRAB}\left(\mathrm{RR}=3,04 ; \mathrm{IC}_{95}\right.$ $1,6-5,6)$ en niños menores de 6 meses comparado con niños entre 18 y 23 meses $^{11}$.

De los factores ambientales como el uso de combustibles fósiles y el tabaquismo intramuros, se ha confirmado ampliamente su efecto en el desarrollo de IRAB en diversos estudios. En particular los combustibles de biomasa, entre ellos la leña, son considerados principal fuente de contaminación del aire de interior ${ }^{12}$. Esto es particularmente importante en zonas donde las fuentes combustibles de uso doméstico como medio de calefacción y cocción de alimentos se sustentan en la utilización de leña, como es en el sur del país. Así se ha reportado una diferencia de su consumo a nivel residencial que supera el $70 \%$ respecto del consumo en la zona central $^{13}$. Comparativamente, el uso de leña genera sustancialmente más emisión contami- nante que otras fuentes alternativas como parafina, gas y electricidad ${ }^{14}$, no obstante, y considerando el precio por unidad calórica, la leña corresponde al insumo energético más barato ${ }^{13}$ lo que justificaría su uso casi universal en las regiones del sur.

Tal como nuestros hallazgos, el tabaquismo pasivo ha sido considerado un importante factor de riesgo de IRA en niños de diferente edad. En lactantes, la exposición a humo de tabaco ambiental dentro del hogar está presente en el aire, las superficies y el polvo ${ }^{15}$. Así, la presencia de tabaquismo en el hogar se ha asociado con un riesgo 2 veces mayor de desarrollar IRAB en lactantes $\left(\mathrm{RR}=2,13\right.$; $\mathrm{IC}_{95} 1,3-$ $3,4)^{11}$. Consecuentemente, la ausencia de TID se ha considerado principal factor protector de hospitalización por bronquiolitis en menores de un año $(\mathrm{OR}=0,54 ; \mathrm{p}=0,0071)^{7}$. En niños sobre los dos años se ha constatado que la exposición más intensa a humo de tabaco ambiental establecida por niveles de cotinina en orina, incrementa ostensiblemente el riesgo de IRAB $\left(\mathrm{OR}=4,7 ; \mathrm{IC}_{95} 2,6-8,5\right)^{16}$. Globalmente, tanto la combustión de sólidos así como el consumo de tabaco en ambientes cerrados son fuentes principales de la contaminación de aire de interior y productores de material particulado (MP). Ante esto los niños poseen una susceptibilidad mayor dada su inmadurez fisiológica y sus características propias de la edad. Así, la exposición a MP en niños pequeños difiere en varios aspectos en relación al adulto y niños mayores, lo que lo expone a una situación particularmente crítica en los primeros meses de vida. Entre estas condiciones se cuentan, la alta relación entre superficie corporal y peso, menor calibre de la vía respiratoria, mayor depósito de partículas en el tracto respiratorio, inmadurez pulmonar e inmadurez inmunológica ${ }^{17,18}$.

Desde el punto de vista estacional, y considerando la etiología viral como predominante en las IRA, estudios previos también describieron el efecto del clima invernal sobre las IRA. La baja temperatura ambiental se ha postulado como un factor coadyuvante en su desarrollo en lactantes, coincidiendo con episodios epidémicos de virus respiratorio sincicial (VRS) e incremento de hospitalizaciones por $\operatorname{IRAB}^{19}$. En Argentina se determinó similarmente la ma- 
yor proporción de casos de hospitalizaciones en menores de 2 años por VRS en invierno ${ }^{20} \mathrm{y}$ en menores de 5 años con IRAB por VRS y virus influenza ${ }^{21}$. Moura et al, describieron un aumento de las IRA y específicamente por VRS en época de mayor precipitación ${ }^{22}$. Por su parte, en España, se ha registrado un aumento de las infecciones por VRS en el periodo invernal, estimándose una significativa correlación negativa de las hospitalizaciones con la humedad y temperatura ${ }^{23}$.

Para las IRAA se ha postulado que el respirar aire frío durante el invierno provoca enfriamiento de la vía aérea nasal generando caída de la temperatura del epitelio respiratorio y disminución de la efectividad de las defensas respiratorias enlenteciendo el clearance mucociliar e inhibiendo la actividad fagocitaria de los leucocitos ${ }^{24}$, de esta manera igualmente se favorecería la llegada y permanencia de los agentes infecciosos en la vía aérea inferior.

La condición conyugal soltera (sin pareja) fue el único factor social asociado significativamente a consultas de morbilidad por IRAB. Hallazgo similar fue reportado en un estudio desarrollado en Australia en que esta condición fue un predictor independiente de infección por $\mathrm{VRS}^{25}$. En dicha investigación esa condición se asoció a menor nivel socioeconómico y mayor exposición a humo de cigarrillo. En nuestro análisis, las evaluaciones estratificadas para distintas variables solo mostraron que las madres que no mantenían pareja actual, presentaron significativa menor proporción de LME al tercer mes, variable que no mostró diferencias en el desarrollo de CME por IRAB. De esta manera, factores aquí no evaluados pudieran ejercer un efecto en relación al estado marital/ conyugal sobre las IRA.

A diferencia de hallazgos previos en que la escolaridad materna fue un factor de riesgo significativo en la enfermedad respiratoria baja ${ }^{26}$, en nuestro estudio no se detectó este efecto. Igualmente esto ocurrió para otros factores socioeconómicos tradicionalmente vinculados al desarrollo de IRAB, lo cual era probable en vista de la poca variabilidad de estas condiciones en el grupo estudiado y recordando que se trata solo de población beneficiaria del sistema público de salud.
Algunas limitaciones de este estudio consideran la pérdida de diagnósticos específicos enunciados como IRA viral alta y en pocos casos de niños con más de una consulta existió dificultad en establecer el término entre un episodio de enfermedad y otro. No obstante creemos que estas situaciones no logran sesgar significativamente nuestros resultados. Por otra parte, la falta de cuantificación de la intensidad de exposiciones como consumo de leña y cigarrillos intradomiciliario impidió que los hallazgos demuestren la fuerza de la asociación centrada en efecto exposición-respuesta sino más bien como una aproximación dicotómica del fenómeno. En este contexto, sería pertinente establecer el comportamiento del consumo de leña y otros combustibles residenciales de manera de definir hogares de mayor riesgo. Asimismo, se deben desarrollar estudios adicionales centrados en cuantificar en detalle los niveles de exposición a estos factores y su efecto en el desarrollo de IRA midiendo la exposición real a contaminantes intramuros a los que están expuestos los lactantes de nuestro medio.

Aun considerando estas limitaciones, este estudio proporciona una estimación actual de la enfermedad respiratoria en el lactante pequeño y la consecuente demanda ejercida a nivel ambulatorio. Da a conocer además la presencia de factores modificables asociados, lo que hace posible considerarlos para concentrar esfuerzos en su modificación, y con ello reducir la incidencia de este todavía importante problema sanitario.

\section{Referencias}

1.- Organización Panamericana de Salud. Investigaciones operativas sobre el control de las infecciones respiratorias agudas (IRA) en Brasil. Washington, DC 1999

2.- López IM, Sepúlveda HB, Guerra JF, Nazar R, Valle P: Perfil de morbilidad anual de menores de 6 años consultantes. Rev Chil Pediatr 2000; 71: 321-7.

3.- Méndez B, Herrera P, Guerra H, Dattas JP, Muñoz B, Velasco $J$ : Estructura de la consulta pediátrica en el Servicio de Urgencia. Hospital Infantil Roberto del Río. Rev Chil Pediatr 2005; 76: 259-65.

4.- López IM, Sepúlveda H, Nazar R, Martínez W, Montero A: Infección respiratoria aguda baja (IRAB) del niño en atención primaria. Rev Chil Pediatr 2001; 72: 204-11 
5.- Ministerio de Salud: Guía clínica infección respiratoria aguda baja de manejo ambulatorio en menores de 5 años. 1 ed. Santiago: Minsal; 2005.

6.- Zar HJ, Mulholland K: Global burden of pediatric respiratory illness and the implications for management and prevention. Pediatr Pulmonol 2003; 36: 457-61.

7.- Zamorano A, Márquez S, Aranguiz JL, Bedregal P, Sánchez I: Relación entre bronquiolitis aguda con factores climáticos y contaminación ambiental. Rev Méd Chile 2003; 131: 1117-22.

8.- Victora CG. Factores de riesgo en las IRA bajas. En: Benguigui Y, López FJ, Schmunis G, Yunis J, editores. Infecciones respiratorias en niños. Washington, DC, OPS 1997; 45-63.

9.- López IM, Sepúlveda H, Valdés I: Acute respiratory illnesses in the first 18 months of life. Rev Panam Salud Publica 1997; 1: 9-17.

10.- Pino P, Walter T, Oyarzún M, Villegas R, Romieu I: Fine particulate matter and wheezing illnesses in the first year of life. Epidemiology 2004; 15: 702-8.

11.- Koch A, Molbak K, Homoe P, et al: Risk factors for acute respiratory tract infections in young Greenlandic children. Am J Epidemiol 2003; 158: 374-84.

12.- Chauhan AJ, Johnston SL: Air pollution and infection in respiratory illness. Br Med Bull 2003; 68: 95-112.

13.- Abalos M: Estudio de casos sobre combustibles forestales. Chile. Proyecto Información y Análisis para el Manejo Forestal Sostenible: Integrando Esfuerzos Nacionales e Internacionales en 13 Países Tropicales en América Latina. Santiago, FAO; 2001.

14.- Smith KR, Samet JM, Romieu I, Bruce N: Indoor air pollution in developing countries and acute lower respiratory infections in children. Thorax 2000; 55: 518-32.

15.- Matt GE, Quintana PJ, Hovell MF, et al: Households contaminated by environmental tobacco smoke: sources of infant exposures. Tob Control 2004; 13: 29-37.
16.- Keskinoglu P, Cimrin D, Aksakoglu G: The impact of passive smoking on the development of lower respiratory tract infections in children. J Trop Pediatr 2007; 53: 319-24.

17.- Schwartz J: Air pollution and children's health. Pediatrics 2004; 113: 1037-43.

18.- Heinrich J, Slama R: Fine particles, a major threat to children. Int J Hyg Environ Health 2007; 210: 617-22.

19.- Avendaño LF, Céspedes A, Stecher X, Palomino MA: Influencia de virus respiratorios, frío y contaminación aérea en la infección respiratoria aguda baja del lactante. Rev Méd Chile 1999; 127: 1073-8.

20.- Videla C, Carballal G, Misirlian A, Aguilar M: Acute lower respiratory infections due to respiratory syncytial virus and adenovirus among hospitalized children from Argentina. Clin Diagn Virol 1998; 10: 17-23.

21.- Viegas $M$, Barrero PR, Maffey AF, Mistchenko AS: Respiratory viruses seasonality in children under five years of age in Buenos Aires, Argentina: a five-year analysis. J Infect 2004; 49: 222-8.

22.- Moura FE, Nunes IF, Silva GB, Jr, Siqueira MM: Respiratory syncytial virus infections in northeastern Brazil: seasonal trends and general aspects. Am J Trop Med Hyg 2006; 74: 165-7.

23.- Lapeña S, Robles MB, Castañón L, et al: Climatic factors and lower respiratory tract infection due to respiratory syncytial virus in hospitalised infants in northern Spain. Eur J Epidemiol 2005; 20: 271-6.

24.- Eccles R: An explanation for the seasonality of acute upper respiratory tract viral infections. Acta Otolaryngol 2002; 122: 183-91.

25.- Reeve CA, Whitehall JS, Buettner PG, Norton R, Reeve $D M$, Francis $F$ : Predicting respiratory syncytial virus hospitalisation in Australian children. J Paediatr Child Health 2006; 42: 248-52.

26.- López IM, Sepúlveda H, Valdés I: Afecciones respiratorias bajas en el lactante: magnitud y factores de riesgo. Rev Chil Pediatr 1994; 65: 154-7. 